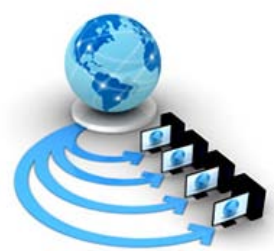

Volume 9, No. 2, March-April 2018

International Journal of Advanced Research in Computer Science

RESEARCH PAPER

\title{
PARTICLE SWARM OPTIMIZATION ALGORITHM (PSO) USED FOR SECURITY ENHANCEMENT IN MANET
}

\author{
S. Mahalakshmi \\ ,M.Sc Project Student \\ Department of Information Technology \\ Bharathiar University \\ Coimbatore-46, India
}

\author{
Dr. R. Vadivel \\ Assistant Professor \\ Department of Information Technology \\ Bharathiar University \\ Coimbatore- 46, India
}

\begin{abstract}
Mobile Ad-hoc NETwork (MANET) has become extremely famous because of its dynamic and infrastructure-less character. MANET contains countless number of mobile nodes which communicate each other in wireless mode. The mobility of nodes in MANET is high when compared to other network, where it does not rely on fixed infrastructure. MANET is frequently changing its topologies to transfer the data quickly, because nodes in this network are moving always (mobility) and data transfer has been done by finding the efficient routing path between source and destination. These types of attacks affect the MANET routing path and it hence it is necessary to secure routing. ALERT protocol is evolved which is Distinguished by its low cost due to randomized routing mechanism and anonymity protection for sources, destinations, and routes. PSO is a population based optimization technique use for finding optimum solution. PSO technique is originated from social behavior bird flocking. In PSO optimum solution is obtained from the behavior of bird. Since PSO uses for network centric localization purpose, this approach generates network navigational decisions by obviating centralized control thereby reducing both the congestion and delay.
\end{abstract}

Keywords: PSO, denial of services, TCP

\section{INTRODUCTION}

Each Sensor Node can contain various sensors and actuators that are used to collect the data and control physical processes. [1] The collected data is transferred to the User through the network that can include Internet segments. The collecting of the data and controlling the node, may need to perform some successive on the measured data.[2] Direct communication between individual nodes can also be required.[3] The Task Manager Node (User) performs tasks in data storage, analysis and display.The wireless sensor nodes for many applications also imply a major portion of these networks would have to acquire self-organization capability. [4] This is most basic wireless network configuration and is the equivalent of a wired peer-to peer mobile network. [5] The range of this configuration is limited and administration sharing an issue with more than just a few nodes. Thus, ad hoc configurations should only be used for the smallest of wireless networks scalability and security are unimportant. Regarding the extensive examination on MANET: (a) it enables the users to understand the benefits of using remote systems (i.e., wireless networks) (b) it empowers the users to choose remote systems for their requirements [6]. Significantly, the above said points obviously distinguishes the general components of remote systems [7]. This empowers general talks on difficulties and arrangements, which can be promptly connected to assorted remote systems. The simplicity of organization without the current framework makes specially appointed systems (i.e., ad hoc networks) an appealing decision for dynamic circumstances, for example, military tasks, catastrophe recuperation, etc. This research work evaluates the case study for mobile Ad Hoc network with concentration to defend against Denial of
Service attack in MANET layers. A military case study scenarios is introduced: the scenario modifies its channel and physical layer settings for army military devices in an unknown and unstable MANET military environment system with concentration to defend against Denial of Service attack

\section{RELATED WORK}

The threshold adjustment and knowledge are random varied. The threshold technique is exploits on the optimal solution. [9]. the threshold schemes used for the wireless local area network. Hence the map is dynamically changed by the threshold policies. The threshold technique is functioned on the threshold compensation on the distributed formulated dynamic changing. The multipath transfer is the estimate congestion control the communication paths are end to end video distortion. [10]. It formulates the data distributed over the transmission theory video peak signals are reduce the noise ratio and packet delay. [11]The end to end distortion is communicated with video streaming the bandwidth iscommunicated on the time varying channels.

The sensor networks are not fully trusted and it arise question of preserving the privacy for the data which was collected and to check the reply send by the receiver from the network [13]. The problem that got raised in the network with context manner is dealt with the nodes which have storage capacity. It is considered as common and dominant query. The concept of bucketization was used to blend the data for a specific range. It also uses encryption for integrity of the data, where it employs encoding of numbers to prevent the storage nodes from dropping data. Believe that pervasive computing systems, touching upon every aspect of life, will be partially supported by the sensor network 
infrastructure, which is involved in two processes: monitoring the environment surrounded and providing information for the analysis and respond. Both processes are exposed to potential risks for information security and privacy prohibiting the realistic sensor network deployment. On one hand, a sensor network may leak information about people to an unauthorized party, which leads to a privacy breaching. Then once again, it might likewise lie about the gathered data to a legitimate inquiry regarding dysfunctional of network. In expanding such a sensible sensor network, there arise an essential inquiry about trusting sensor network, that is, how to prohibit the detection of misbehaviour. Lamentably, tiny research work has focused to take care of the issue. This paper tries to address the problem in a setting of network enhanced by some nodes with large storage space and considers a powerful and typical sensor network operation: range query. The Storage nodes are expected to be placed as intermediate tier of large scale sensor networks. An important issue is compromised storage node may not only cause the privacy problem, but also return fake incomplete query results. The middle tier is composed of a small number of storage-abundant nodes, called storage nodes. The bottom tier consists of a large number of resource-constrained ordinary sensors that sense the environment.

\section{METHODOLOGY}

\section{A. MANET Construction}

MANET-satellite scenario, high latency, bandwidth asymmetry, and transmission errors on wireless channels remarkably affect the TCP performance. Regarding the terrestrial wireless hop, channel losses due to (time-varying) multipath fading may be dominant (or comparable to) congestion losses. TCP standard mechanisms significantly improved the performance of long-lived connections with multiple random losses in the same window of data. New Reno addresses this problem by introducing partial acknowledgments in the loss recovery.

\section{B. Data Transfer Between Source To Destination:}

The node is treated on the data communication equipment. The data terminal point is connected on the source and destination on the intermediate source of data transmission. This is a functionality of creating data or receiving information in communication channel. Examples of the Data Communication equipment or Nodes can be Modem, Hub, Switch, bridge. Data Terminal Equipment or Node can be Router or Host Parallel multipath video streaming.

\section{Alert Protocol}

ALERT can be applied to different network models with various node movement patterns such as random way point model and group mobility model. It is necessary to consider a MANET which is deployed in vast field; the type of routing used for communication of node is geographic with a specific end goal to lessen the correspondence idleness. Sender location uncovered by simply bares the direction of transmission. By analyzing, it is getting an conclusion that it is necessary an mysterious protocol which can make the sender untraceable from other nodes.

\section{Path Selection Using (PSO)}

The Particle Swarm Optimization Algorithm (PSO) used to reduce the number of iteration in the $\mathrm{k}$ means ++ algorithm. The PSO algorithm eliminates the semantic gap between the features in order to reduce the query time and precision of retrieval results. The PSO is capable to retrieving the data from the class which are even not found the training phase as it is works with huge work space. The Experimental results proves that novel proposed mechanism outperforms the state of art approaches in terms of precision ,recall and $\mathrm{f}$ measures values which indicates the accuracy also in execution time which indicates the efficiency. In the feature selection using PSO algorithm the entropy value is calculated.

\section{SIMULATION ENVIRONMENT}

This project is been developed using Java EE and Java Net beans environment. Net beans IDE enables the user to rapidly and effectively create applications for desktop, mobile and web along with HTML5 applications with HTML, JavaScript and CSS. It has extensive group of clients and engineers all around the globe due to its open source.

Preeminent Support for Current Java Technologies

The authority IDE for Java 8 is Net Beans. The user can develop an applications rapid and easy manner due to its editors, analyzer of code and converters. The user can redesign or update their applications to Java 8 to use lambdas, functional operations, and method references.

\section{Quick and Smart Code Editing}

Net Beans IDE is significantly more than a ordinary editor. The Editor in Net Beans align lines, matches words and sections, and features the source code syntactically and semantically. It allows effortlessly refractor code, with a scope of convenient and intense tools, where it additionally gives code formats, tips for coding, and code generators.

\section{Simple Management of Efficient Project:}

Keeping in mind about large applications, with great many files, folders and lines of code, is an overwhelming task. It provides diverse perspectives of the information from numerous projects. It gives the user a chance to penetrate down into the information rapidly and effectively.

Write Bug Free Code

The code which has bugs no-longer stays unfixed. Net Beans gives static examination of tools, particularly incorporation with the generally utilized tool namely Find Bugs.

Simulation Requirements

\begin{tabular}{|l|l|l|l|l|}
\hline \multicolumn{2}{|l|}{ Software requirements } & \multicolumn{2}{|l|}{ Hardware requirements } \\
\hline IDE & Net beans & Processor & $\begin{array}{l}\text { 800MHz Intel } \\
\text { Pentium III or } \\
\text { equivalent }\end{array}$ \\
\hline $\begin{array}{l}\text { Developer } \\
\text { tool }\end{array}$ & $\begin{array}{l}\text { Java SE } \\
\text { Development } \\
\text { Kit (JDK) }\end{array}$ & Memory & $350 \mathrm{~GB}$ \\
\hline $\begin{array}{l}\text { Operating } \\
\text { system }\end{array}$ & Windows & RAM & $1 \mathrm{~GB}$ \\
\hline
\end{tabular}




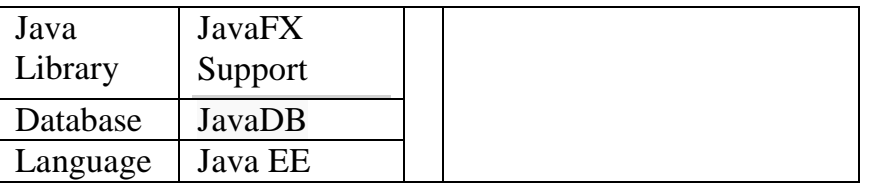

\section{PERFORMANCE EVALUATION}

In this result dynamic path strategy, and PSO algorithm experiments like execution time, throughput is calculated. The PSO execution strategy was less time consumed.

Table 1: Performance Metrics

\begin{tabular}{|c|c|c|}
\hline Algorithm Name & Metrics & Value \\
\hline $\begin{array}{c}\text { Dynamic Path } \\
\text { Strategy }\end{array}$ & $\begin{array}{c}\text { Execution } \\
\text { Time }\end{array}$ & 12226 \\
\hline PSO & $\begin{array}{c}\text { Execution } \\
\text { Time }\end{array}$ & 7925 \\
\hline $\begin{array}{c}\text { Dynamic Path } \\
\text { Strategy }\end{array}$ & Throughput & 1.2 \\
\hline PSO & Throughput & 2.5 \\
\hline
\end{tabular}

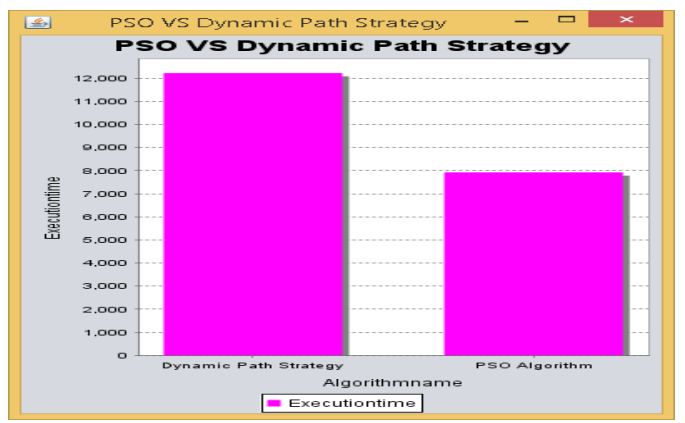

Fig 1: Dynamic Strategy path strategy has been compared to PSO path Strategy.

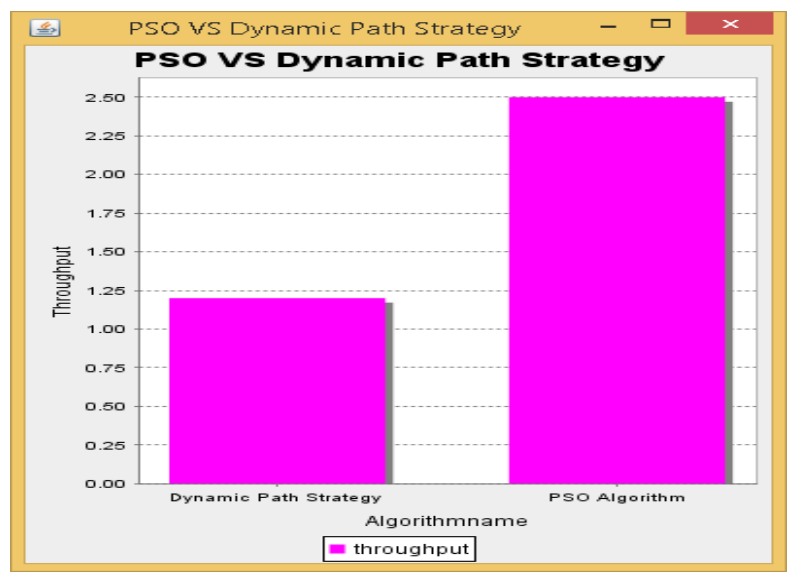

Fig 2: Dynamic Strategy path strategy has been compared to PSO path Strategy

\section{CONCLUSION}

The proposed approach prolongs the lifetime of the WSN system. In case of dying of some nodes, system can automatically construct new paths for distribute the data. An experimental work is conducted to measure the performance of the system in terms of estimating the PSO path selection for improve throughput and reduce less time.

\section{REFERENCES}

[1] Peter Desnoyers, Deepak Ganesan, et al., "PRESTO: A Predictive Storage Architecture for Sensor Networks" University of Massachusetts Amherst, Conference: Proceedings of HotOS'05: 10th Workshop on Hot Topics in Operating Systems, June 12-15, Santa Fe, New Mexico, USA, 2005.

[2] Bo Sheng and QunLi, "Verifiable Privacy-Preserving Range Query in Two-tiered Sensor Networks”, US National Science Foundation award CCF-0514985 and CNS0721443, 2006.

[3] B. Sheng, Q. Li, "Verifiable privacy-preserving range query in two-tiered sensor networks", 27th Conference on Computer Communications, IEEE, 2008, doi: 10.1109/INFOCOM.2008.18.

[4] Bijit Hore, Sharad Mehrotra, Gene Tsudik "A PrivacyPreserving Index for Range Queries”, Proceedings of the 30th VLDB Conference, Toronto, Canada, 2004, p.g: 720-733

[5] Chia-Mu Yu, Guo-Kai Ni, “Top-K Query Result Completeness Verification in Tiered Sensor Networks' IEEE Transactions on Information Forensics and Security, Vol. 9, No. 1, January 2014, p.g: 109-124

[6] Rui Zhang, jing Shi et al, "Top-k Query Processing in Unattended Tiered Sensor Networks Rui Zhang”, IEEE Transactions on Vehicular Technology, 2014, p.g: 18-22.

[7] Demetrios Zeinalipour-Yazti, et al "Micro Hash: An Efficient Index Structure for Flash-Based Sensor Devices DemetriosZeinalipour-Yazti”, 2012, FAST '05: 4th USENIX Conference on File and Storage Technologies, p.g: 31-44.

[8] Muhammad Naveed, Seny Kamara, “Inference Attacks on property-Preserving Encrypted Databases”, ACM 978-14503-3832, doi:10.1145/2810103.2813651.

[9] Jing Shi, Rui Zhang, and Yanchao Zhang, "Secure Range Queries in Tiered Sensor Networks", Proceedings - IEEE INFOCOM, 2009, p.g: 945- 953.

[10] Xiaoping Liao ,Jianzhong Li, “Privacy-preserving and Secure Top-k Query in Two-tier Wireless Sensor Network",IEEE Global communications conference, doi: 10.1109/GLOCOM.2012.6503135, 2009.

[11] W. Adjie-Winoto, E. Schwartz, and H. Balakrishnan, "The Design and Implementation of an Intentional Naming System”, 17th ACM Symposium on Operating Systems Principles, Published as Operating Systems Review, 34(5):186-201, Dec. 1999.

[12] P. Bose, P. Morin, I. Stojmenovi'c, and J. Urrutia, "Routing with Guaranteed Delivery in Ad Hoc Wireless Networks", In Workshop on Discrete Algorithms and Methods for Mobile Computing and Communications (DialM '99), Seattle, Washington, August 1999, p.g: 48-55.

[13] N. Bulusu, J. Heidemann, and D. Estrin, "GPS-Less Low Cost Outdoor Localization for Very Small Devices”, IEEE Personal Communications Magazine, Special Issue on Smart Spaces and Environments, October2000,

[14] A. Cerpa, J. Elson, D. Estrin, L. Girod, M. Hamilton, and J. Zhao, "Habitat Monitoring: Application Driver for Wireless Communications Technology", ACMSIGCOMM Workshop on Data Communications in Latin America and the Caribbean, Costa Rica, April 2001, p.g: 20-41, doi: 10.1145/371626.371720. 